\title{
EFFECTS OF EXTERNAL MAGNETIC FIELDS ON THE PHOTOELECTRON EMISSION FROM A COPPER BEAM CHAMBER
}

\author{
Y. Suetsugu, Y. Tanimoto, Y. Hori, K. Kanazawa and M. Kobayashi, KEK, Tsukuba, Japan \\ Y-J. Hsu, SRRC, Hsinchu, Taiwan, R.O.C.
}

\begin{abstract}
Applying magnetic fields around a beam chamber seems to be one of the effective methods to suppress the generation of electron cloud due to photoelectrons and, therefore, the beam size blow up of the positron beam observed at the KEKB. In order to investigate the effective magnetic field configuration and to estimate the necessary strength of it, the photoelectron current at the center of a test chamber is measured applying external magnetic fields. The test chamber is made of copper as the real beam chamber. The external magnetic fields are applied by a solenoid or small pieces of permanent magnets changing the magnetic field configuration, the structure of irradiated surface and the bias voltage of electrodes. The uniform magnetic field larger than about $50 \mathrm{G}$ is found to be useful to reduce the photoelectrons at the beam position.
\end{abstract}

\section{INTRODUCTION}

To overcome the beam size blow up of the positron beam is one of the most important issue for KEKB at present to improve the luminosity. Many simulations indicated that the blow up is due to the electron cloud around the beam [1]. This cloud would excite a head-tail type instability of the beam. The seed of the electron cloud is the photoelectrons emitted from the chamber surface irradiated by the synchrotron radiation (SR).

The previous experiment to estimate the photoelectron yield using a copper test chamber showed that the photoelectrons emitted from the surface have an energy of less than $5 \mathrm{eV}$ at most [2]. The magnetic field near to the surface, therefore, can prevent photoelectrons from going to the center (beam position) of the beam chamber. A magnetic field produced by small pieces of permanent magnets or solenoids, therefore, can be a useful method to suppress the blow up of the positron beam at relatively little expense.

The purpose of this study is to investigate the effect of a magnetic field on the number of photoelectrons at the beam position. The photoelectron current of electrode located at the center of the test chamber was measured for various magnetic field configurations generated by permanent magnets or solenoids. The measurements were also performed for the smooth surface and the sawtooth surface [2]. For some cases, a positive potential was applied on the electrode to assume the positron beam.

\section{EXPERIMENTAL SET UP}

The BL-21 beam line at PF (Photon Factory) at the KEK was used for the study. Basically, the experimental set up, the parameters of the SR and the structure of test chamber are the same as the previous experiment [2]. The critical energy of SR is $4.1 \mathrm{keV}$. The inside structure of the test chamber is shown in Figure 1. The test chamber is made of copper. The diameter and the length are $94 \mathrm{~mm}$ and $300 \mathrm{~mm}$, respectively. The SR irradiates the chamber surface at a mean incidence angle of 3 degrees $(52 \mathrm{mrad})$. The irradiated length is about $100 \mathrm{~mm}$. In order to see the spatial photoelectron distribution, 15 copper electrodes (12 mm x $30 \mathrm{~mm})$, five rows axially (No.1- No.5) and three lines azimuthally $(\mathrm{A}, \mathrm{B}, \mathrm{C})$, are arranged above the irradiated surface. Here we focused on the photoelectron current at the center of the chamber, that is, the current at the electrodes of A line (from A-1 to A-5). The photoelectron current, $I_{p}$, is defined by $I_{p}=I_{m}(0 \mathrm{~V})-I_{m}(-11 \mathrm{~V})$, where $I_{m}(0 \mathrm{~V})$ and $I_{m}(-$ $11 \mathrm{~V})$ are the measured current, $I_{m}$, at a bias voltage of $0 \mathrm{~V}$ and $-11 \mathrm{~V}$, respectively.

The permanent magnet is made of Ferrite. The diameter and the thickness are $30 \mathrm{~mm}$ and $8 \mathrm{~mm}$, respectively. The residual magnetic field is $1.2 \mathrm{kG}$ on the surface. The magnets are attached from outside of the chamber as shown in Figure 1, for an example.

The solenoid field is made by winding $0.25 \mathrm{~mm}^{2}$ power cable around the test chamber. The total solenoid length was about $130 \mathrm{~mm}$. A typical axial magnetic field just near the surface is about $20 \mathrm{G}$ at the solenoid current of 5 A.

\section{RESULTS AND DISCUSSIONS}

\subsection{6 permanent magnets}

Figure 1 and 2 show the arrangements of the 16 permanent magnets and their 4 configurations. The axial space between the adjacent magnets is $50 \mathrm{~mm}$. Azimuthally (normal to the axis), there are two configurations, $\{B\}$ and $\{Q\}$. The $\{B\}$ means the magnetic field inside the chamber is similar to that of a bending magnet. The $\{\mathrm{Q}\}$ means, on the other hand, the magnetic field is similar to that of a quadrupole magnet. Axially, there is also two variations, $\{\mathrm{NNNN}\}$ and $\{N S N S\}$. The $\{N N N N\}$ means the polarity of magnets are the same for 4 magnets aligned axially. The $\{$ NSNS $\}$ means the polarity changed alternatively on the contrary. The magnetic field just near a magnet piece is about $50 \mathrm{G}$ at the inner surface.

The axial distributions of $I_{p} / I_{b}$ for these 4 magnetic field configurations are shown in Figure 3 , where the $I_{b}$ is the stored beam current. The dotted line is that for the case without magnet. The irradiated surface is a smooth surface [2]. The peak $I_{p} / I_{b}$ among A-1 - A-4 electrodes for 4 magnetic field configurations are compared in the 

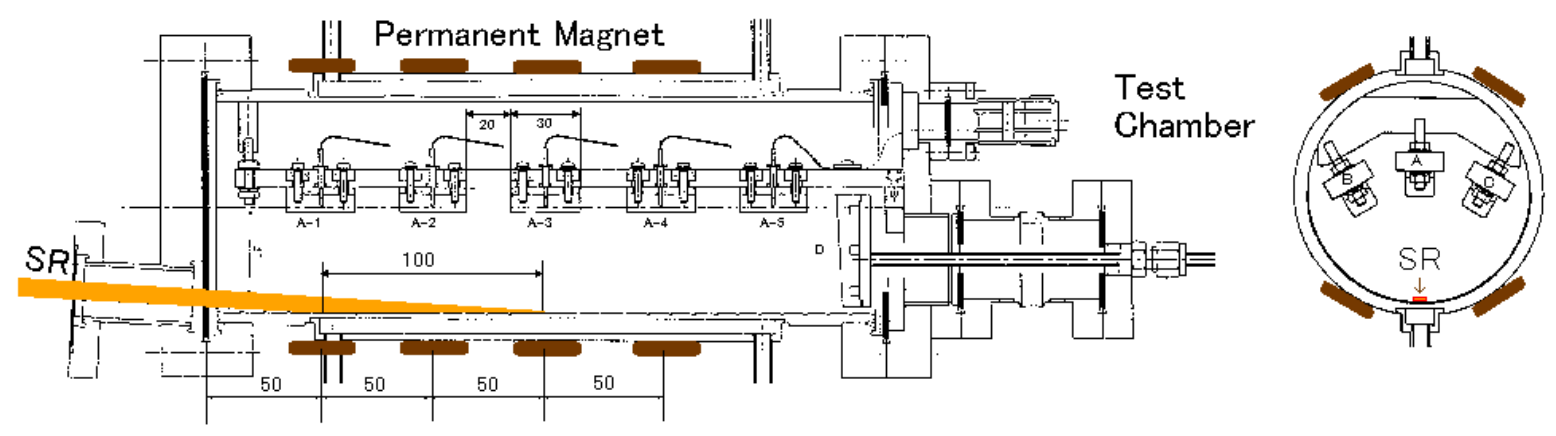

Figure 1:Inside structure of the test chamber and the setting of 16 permanent magnets around it.

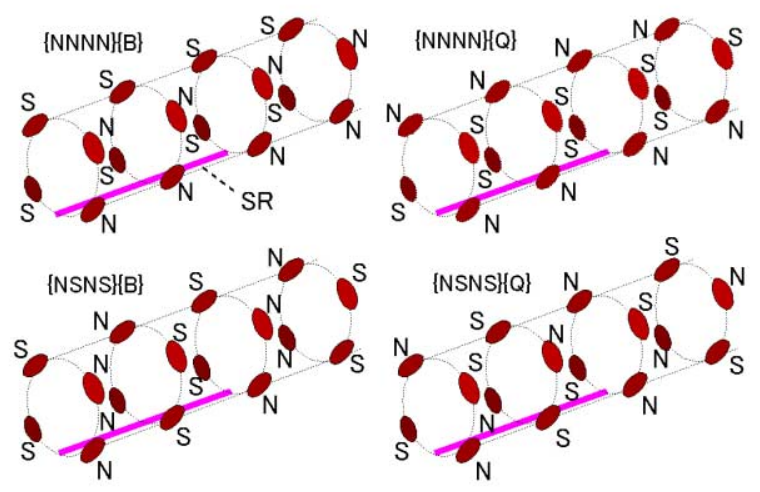

Figure 2: Four configurations of 16 permanent magnets. See the text for the meanings of $\{\mathrm{NNNN}\}$, $\{\mathrm{B}\}$ and so on.

Table 1. The uniform magnet field, $\{\mathrm{NNNN}\}$, is more effective than the alternative configuration, $\{\mathrm{NSNS}\}$, to reduce the photoelectron current. Furthermore, the bending magnetic field, $\{\mathrm{B}\}$, is better than the qudrupole configuration, $\{\mathrm{Q}\}$. The case of $\{\mathrm{NNNN}\}\{\mathrm{B}\}$, the peak $I_{p} / I_{b}$ is less than $0.6 \%$ of the value without magnet. The reduction is, on the contrary, about $30 \%$ at most for the case $\{$ NSNS $\}$. The results indicate that the uniform magnetic field is important to reduce the photoelectrons at the beam position.

\subsection{8 permanent magnets}

Similarly to the previous case, the four magnetic field configurations were arranged using 8 permanent magnets. In this case the axial space between the adjacent magnets is $100 \mathrm{~mm}$. The magnets were fixed axially near the A-1 and A-3 electrode positions. The results are also presented in Table 1. The symbols $\{\mathrm{NN}\}$ and $\{\mathrm{NS}\}$ mean the configurations of uniform and alternative magnet direction as before. The reduction of $I_{p} / I_{b}$ is much less than the case for 16 magnets case except for the case $\{\mathrm{NN}\}\{\mathrm{B}\}$. That may be due to the weak and nonuniform magnetic field compared to the previous case, especially at the A-2 electrode position (between the axially adjacent magnets).

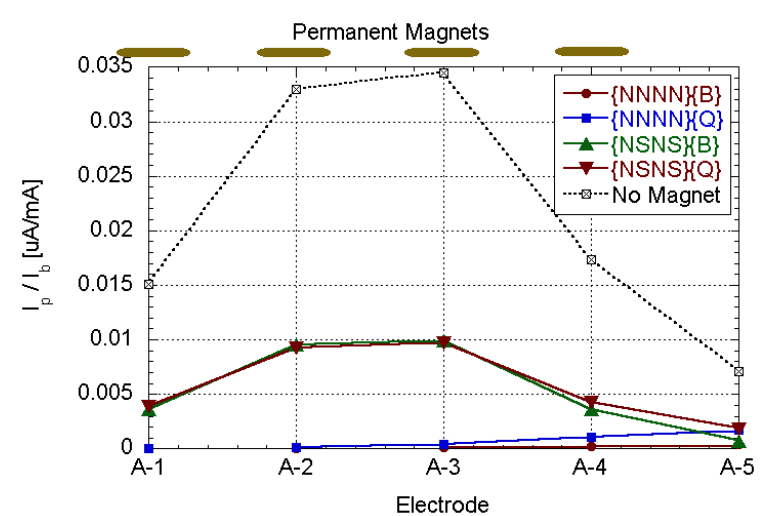

Figure 3: Results of $I_{p} / I_{b}$ for four configurations by 16 permanent magnets in Figure 2.

Table 1: Summary of results for permanent magnets.

\begin{tabular}{c|c:c}
\hline \hline Configuration & $\begin{array}{c}\text { Peak } I_{p} / I_{b} \\
(\mathrm{~A}-1-\mathrm{A}-3)\end{array}$ & $\begin{array}{c}\text { Ratio to } \\
\text { No magnet }\end{array}$ \\
\hline \hline No magnet & 0.0345 & 1 \\
$\{\mathrm{NNNN}\}\{\mathrm{B}\}$ & 0.000196 & 0.00568 \\
$\{\mathrm{NNNN}\}\{\mathrm{Q}\}$ & 0.00103 & 0.0299 \\
$\{\mathrm{NSNS}\}\{\mathrm{B}\}$ & 0.00993 & 0.288 \\
$\{\mathrm{NSNS}\}\{\mathrm{Q}\}$ & 0.00973 & 0.282 \\
\hline$\{\mathrm{NN}\}\{\mathrm{B}\}$ & 0.00715 & 0.0507 \\
$\{\mathrm{NN}\}\{\mathrm{Q}\}$ & 0.0174 & 0.504 \\
$\{\mathrm{NS}\}\{\mathrm{B}\}$ & 0.0197 & 0.571 \\
$\{\mathrm{NS}\}\{\mathrm{Q}\}$ & 0.0226 & 0.655 \\
\hline \hline
\end{tabular}

\subsection{Uniform solenoid field}

As a next step a solenoid was winded around the test chamber and a uniform solenoid field was applied. The axial distributions of $I_{p} / I_{b}$ for three magnetic field strengths, $30 \mathrm{G}, 60 \mathrm{G}$ and $90 \mathrm{G}$, are presented in Figure 4. The peak values of $I_{p} / I_{b}$ among the A-1 - A-3 electrodes are summarized in Table 2. For the magnetic field larger than $60 \mathrm{G}$, the reduction is almost same as the case of the 


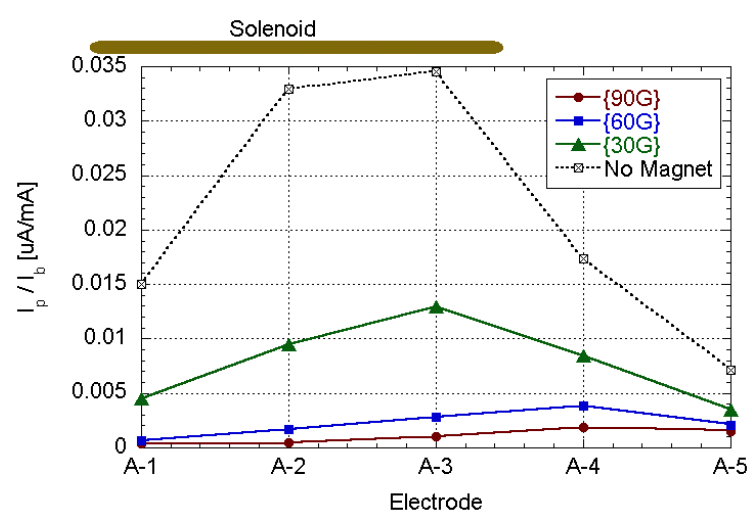

Figure 4: Results of $I_{p} / I_{b}$ for a solenoid field, where the magnetic field is uniform along the solenoid. The solenoid position relative to the electrodes is shown at the upper side.

permanent magnets of $\{\mathrm{NNNN}\}$ in Sec.3.1. On the view point of making a uniform magnetic filed, however, the solenoid field is easier than that of permanent magnets.

\subsection{Alternative solenoid field}

Here the previous solenoid was divided to a half and the alternative solenoid field was generated. The length of each solenoid is about $65 \mathrm{~mm}$. The magnetic field changes oppositely at the axial position of A-2 electrode. The peak $I_{p} / I_{b}$ is presented in Table 2 with a symbol of \{Alt \}. As expected the $I_{p} / I_{b}$ is only a half even for the magnetic field of $60 \mathrm{G}$ compared to the case of no magnets. The peak was recorded at the A-2 electrode.

\subsection{Effect of saw-tooth surface}

The saw-tooth surface was found to be effective to reduce the photoelectron yield [2]. Here the uniform solenoid field was combined to the saw-tooth surface. The results are also listed in Table 2 with a symbol of \{Saw . Even without the magnetic field the $I_{p} / I_{b}$ is about $7 \%$ of the case of smooth surface. By applying the solenoid field, the reduction becomes much effective and reduce to $0.3 \%$ at $60 \mathrm{G}$.

\subsection{Effect of positive potential}

The measurements so far have been performed in the condition that the electrode is at the ground potential. Actually, the positron beam produces the positive potential around the beam and attracts the photoelectrons emitted from the surface. So the measurement was also performed applying $+70 \mathrm{~V}$ on the electrode for the smooth and the saw-tooth surfaces. The results are shown in Table 2 with a symbol of $\{+70 \mathrm{~V}\}$.

By applying $+70 \mathrm{~V}$, the $I_{p} / I_{b}$ increased by one order of magnitude compared to the ground bias case without magnetic field for both surfaces. With the external solenoid field of $42 \mathrm{G}$, however, the $I_{p} / I_{b}$ decreased to less than about $6 \%$ of the case without solenoid filed for both surfaces. That means the external solenoid field will be
Table 2: Summary of results with solenoid.

\begin{tabular}{c|c:c}
\hline Configuration & $\begin{array}{c}\text { Peak } I_{p} / I_{b} \\
(\mathrm{~A}-1-\mathrm{A}-4)\end{array}$ & $\begin{array}{c}\text { Ratio to } \\
\text { No magnet }\end{array}$ \\
\hline \hline No Magnet & 0.0345 & 1 \\
$\{30 \mathrm{G}\}$ & 0.0129 & 0.373 \\
$\{60 \mathrm{G}\}$ & 0.00284 & 0.0823 \\
$\{90 \mathrm{G}\}$ & 0.000992 & 0.0288 \\
\hline$\{\mathrm{Alt}\}\{20 \mathrm{G}\}$ & 0.0289 & 0.838 \\
$\{\mathrm{Alt}\}\{40 \mathrm{G}\}$ & 0.0226 & 0.655 \\
$\{\mathrm{Alt}\}\{60 \mathrm{G}\}$ & 0.0175 & 0.507 \\
\hline$\{\mathrm{Saw}\}\{0 \mathrm{G}\}$ & 0.00231 & 0.0670 \\
$\{$ Saw $\}\{30 \mathrm{G}\}$ & 0.000425 & 0.0123 \\
$\{$ Saw $\}\{60 \mathrm{G}\}$ & 0.000108 & 0.00313 \\
\hline$\{+70 \mathrm{~V}\}\{0 \mathrm{G}\}$ & 0.242 & $7.014(1)$ \\
$\{+70 \mathrm{~V}\}\{42 \mathrm{G}\}$ & 0.0136 & $0.394(0.056)$ \\
\hline$\{$ Saw $\}\{+70 \mathrm{~V}\}\{0 \mathrm{G}\}$ & 0.0224 & $0.649(1)$ \\
$\{$ Saw $\}\{+70 \mathrm{~V}\}\{42 \mathrm{G}\}$ & 0.00101 & $0.029(0.045)$ \\
\hline \hline
\end{tabular}

effective even in the actual case where the positron beam is passing. Note, however, that the applied voltage here is DC and the condition is not completely same as the real case.

\section{SUMMARY}

The external magnetic field was found to be useful to reduce the photoelectrons at the beam position. The uniformity of field was important to suppress the electrons effectively. The magnetic field larger than $50 \mathrm{G}$ reduce the photoelectrons to less than $10 \%$ at the beam position. The magnetic field was also effective even with a positive potential.

Until now, the solenoids were actually winded around the LER beam chamber for about $1200 \mathrm{~m}$ out of $3100 \mathrm{~m}$ of the KEKB ring. The strength is about $45 \mathrm{G}$ just near one solenoid. The solenoid field worked effectively to reduce the blow up of the beam size [3].

\section{REFERENCES}

[1] H.Fukuma et al., "Observation of vertical beam blowup in KEKB Low Energy Ring", Proceedings of EPAC 2000, Vienna, p.1122.

[2] Y.Suetsugu et al., "REDUCTON OF THE PHOTOELECTRON YIELD FROM A COPPER BEAMCHAMBER BY SAW-TOOTH MACHINING", this proceedings.

[3] H.Fukuma et al., "STUDY OF VERTICAL BEAM BLOWUP IN KEKB LOW ENERGY RING", Proceedings of HEACC2001, Tsukuba. 